\title{
Erratum: Memory effect and non-Markovian dynamics in an open quantum system [Phys. Rev. A 99, 052119(2019)]
}

Fujing Liu, Xing-Xiang Zhou, and Zheng-Wei Zhou

(Received 12 June 2019; published 2 July 2019)

DOI: 10.1103/PhysRevA.100.019901

There was an error in Eq. (A17) in the Appendix of the original paper. Specifically, the term $\left|x_{k_{2}}\right|^{2} \cos ^{2} \theta_{k_{2}} e^{-2 i \epsilon_{k_{2}}(t-\tau)} 4\left|y_{k_{2}}\right|^{2} \cos ^{2} \theta_{k_{2}}$ should have been $\left|x_{k_{2}}\right|^{2} \sin ^{2} \theta_{k_{2}} e^{-2 i \epsilon_{k_{2}}(t-\tau)}+4\left|y_{k_{2}}\right|^{2} \cos ^{2} \theta_{k_{2}}$. The following is the correct form for Eq. (A17) in its entirety:

$$
\begin{aligned}
C_{2}(t, \tau)= & \left\langle\varphi(\tau)\left|e^{i H_{E}(t-\tau)} m e^{-i H_{E}(t-\tau)} m\right| \varphi(\tau)\right\rangle \\
= & \left\langle\varphi_{4} \mid \varphi_{3}\right\rangle \\
= & 4 \sum_{k_{2}>0}\left[\sum _ { \substack { k _ { 3 } > 0 \\
k _ { 3 } \neq k _ { 2 } } } \prod _ { k \neq k _ { 2 } , k _ { 3 } } ( | x _ { k } | ^ { 2 } + | y _ { k } | ^ { 2 } ) \left(x_{k_{2}} y_{k_{2}}^{*} e^{2 i \epsilon_{k_{2}}(t-\tau)} \sin \theta_{k_{2}}\right.\right. \\
& \left.+x_{k_{2}}^{*} y_{k_{2}} \sin \theta_{k_{2}} e^{-2 i \epsilon_{k_{2}}(t-\tau)}+2\left|y_{k_{2}}\right|^{2} \cos \theta_{k_{2}}\right)\left(x_{k_{3}}^{*} y_{k_{3}} \sin \theta_{k_{3}}+x_{k_{3}} y_{k_{3}}^{*} \sin \theta_{k_{3}}+2\left|y_{k_{3}}\right|^{2} \cos _{k_{3}}\right) \\
& +\prod_{\substack{k>0 \\
k \neq k_{2}}}\left(\left|x_{k}\right|^{2}+\left|y_{k}\right|^{2}\right)\left(\left|y_{k_{2}}\right|^{2} e^{2 i \epsilon_{k_{2}}(t-\tau)} \sin ^{2} \theta_{k_{2}}+\left|x_{k_{2}}\right|^{2} \sin ^{2} \theta_{k_{2}} e^{-2 i \epsilon_{k_{2}}(t-\tau)}+4\left|y_{k_{2}}\right|^{2} \cos ^{2} \theta_{k_{2}}\right. \\
& \left.\left.+2 x_{k_{2}}^{*} y_{k_{2}} \sin \theta_{k_{2}} \cos \theta_{k_{2}} e^{-2 i \epsilon_{k_{2}}(t-\tau)}+2 x_{k_{2}} y_{k_{2}}^{*} \sin \theta_{k_{2}} \cos \theta_{k_{2}}\right)\right] .
\end{aligned}
$$

Because of this correction in Eq. (A17), Fig. 3(b) in the original paper needs to be updated slightly as follows. There is no material change in the figure's characteristics that are relevant to the physics studied in the paper, however. All conclusions of the paper remain unchanged.

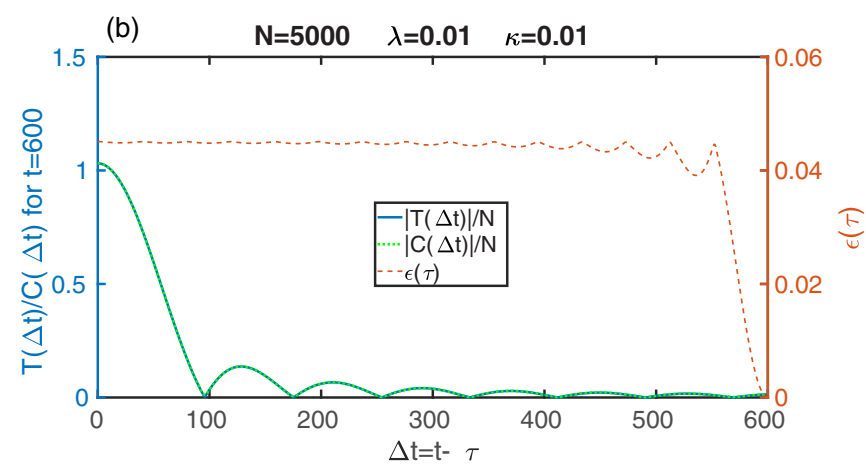

FIG. 3(b). The memory effect term $T(\Delta t)$, the time correlation of the environment $C(\Delta t)$, the system-environment entanglement $\epsilon(\tau)$ against the time-interval $\Delta t$ when the field strength is close to $\lambda=0$ for $t=0$ and $N=5000$. 